\title{
EPR signal reduction kinetic of several nitroxyl derivatives in blood in vitro and in vivo
}

\author{
Zhivko Zhelev ${ }^{1}$, Ken-Ichiro Matsumoto ${ }^{2}$, Veselina Gadjeva ${ }^{3}$, Rumiana Bakalova ${ }^{1}$, Ichio Aoki ${ }^{1}$, \\ Antoaneta Zheleva ${ }^{3}$ and Kazunori Anzai ${ }^{2}$ \\ ${ }^{1}$ Department of Biophysics, Molecular Imaging Center, National Institute of Radiological Sciences, Chiba, Japan; \\ E-mail: zh_zhelev@yahoo.com \\ ${ }^{2}$ Heavy-ion Radiology Research Center, National Institute of Radiological Sciences, 4-9-1 Anagawa, Inage-ku, Chiba 263-8555, Japan \\ ${ }^{3}$ Department of Chemistry and Biochemistry, Trakia University, Stara Zagora, Bulgaria
}

\begin{abstract}
The present study is focused on the mechanism(s) of electron-paramagnetic resonance (EPR) signal reduction kinetic of several nitroxyl radicals and nitroxyl-labeled anticancer drugs in physiological solutions in the context of their application for evaluation of oxidation/reduction status of blood and tissues - an important step in biomedical diagnostics and planning of therapy of many diseases. The nitroxyl derivatives were characterized with different size and water-solubility. Some of them are originally synthesized. In buffer, in the absence of reducing and oxidizing equivalents, the EPR signal intensity of all nitroxyls was constant with the time. In serum and cell cultured medium, in an absence of cells and in a negligible amount of reducing and oxidizing equivalents, there was no significant EPR signal reduction, too. In vitro (in freshly isolated blood samples), the EPR signal intensity was characterized with slow decrease within $30 \mathrm{~min}$, presumably as a result of interaction between the nitroxyl derivative and blood cells. The EPR spectrum of hydrophobic nitroxyls showed a slight anisotropy in cell-containing solutions and it did not changed in non-cell physiological solutions. This suggests for a limited motion of more hydrophobic nitroxyls through their preferable location in cell membranes. In vivo (in the bloodstream of mice under anesthesia), the EPR signal reduction kinetic was characterized by two phases: i) a rapid enhancement within $30 \mathrm{~s}$ as a result of increasing of nitroxyl concentration in the bloodstream after its intravenous injection, followed by ii) a rapid decrease ( $80-100 \%)$ within $2-5 \mathrm{~min}$, presumably as a result of transportation of nitroxyl in the tissues. The hydrophobic nitroxyls were characterized with stronger and faster decrease in EPR signal intensity in the blood in vivo, as a result of their higher cell permeability, rapid clearance from the bloodstream and/or transportation in the surrounding tissues. The hydrophilic nitroxyls persist in the bloodstream (in their radical form) for a comparatively long time.

The data suggest that the hydrophobic cell-permeable nitroxyl derivatives are most appropriate for evaluation of cell and tissue oxidation/reduction status, while the hydrophilic nitroxyls (impermeable for cell membranes or with very slow cell permeability) are most appropriate for evaluation of oxidation/reduction status of blood using EPR imaging.
\end{abstract}

Key words: Nitroxyl radicals — Nitroxyl-labeled nitrosoureas — EPR — Blood — Imaging

\section{Introduction}

The nitroxyl radicals are well-known from electron-paramagnetic resonance (EPR) studies (Valgimigli et al. 2001;

Correspondence to: Zhivko Zhelev, Department of Biophysics, Molecular Imaging Center, National Institute of Radiological Sciences, 4-9-1 Anagawa, Inage-ku, Chiba 263-8555, Japan E-mail: zh_zhelev@yahoo.com
Kuppusamy et al. 2002; Hirayama et al. 2003; Yokoyama et al. 2004). They are small molecules, sensitive to redox status of biological samples (Fig. 1) and are usually used for tissue oxygen and redox mapping in vitro and in vivo (Valgimigli et al. 2001; Kuppusamy et al. 2002; Hirayama et al. 2003; Yokoyama et al. 2004; Hyodo et al. 2006; Matsumoto et al. 2006; Utsumi et al. 2006). The paramagnetic nitroxyl radical could be reduced to diamagnetic hydroxylamine with loss of EPR signal and thus to serve as reduction sensor. However, 


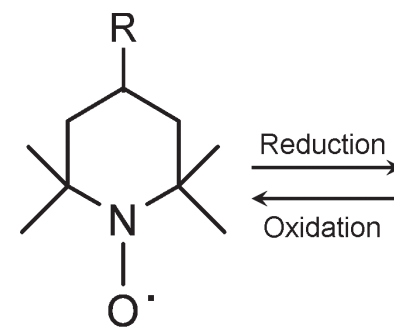

Oxidized form (nitroxyl radical) EPR contrast

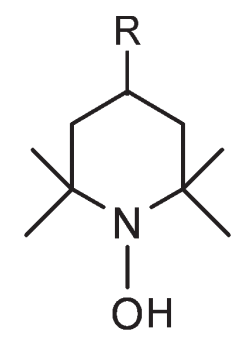

Reduced form (hydroxylamine) Non-contrast
Figure 1. Stable nitroxyl radicals as reduction and oxidation sensors.

the diamagnetic hydroxylamine could be re-converted via oxygenation to paramagnetic nitroxyl radical with appearance of EPR signal and thus to serve as oxidation sensor. The rate-constants of both processes could be used for evaluation of reduction/oxidation balance in cells and tissues.

Using EPR imaging, a very good correlation has been found between the level of oxygen and rate constant of nitroxyl reduction, as well as between the level of reducing equivalents and rate constant of nitroxyl oxidation (Matsumoto et al. 2007; Hyodo et al. 2008). Thus, the balance between the reduction activity of biological fluids or tissues and local oxidation reactions determines the rate of EPR signal decay of nitroxyl radical. In this context, the nitroxyl radicals could be considered appropriate chemical sensors for evaluation of reduction potential and oxidation status of biological specimens using EPR.

The evaluation of reduction potential and oxidation status of blood and tissues (redox and oxygen mapping) has a large prognostic value for treatment of many diseases, accompanied with a significant reduction/oxidation dis-balance, e.g. cancer, neurodegenerative diseases, atherosclerosis, inflammation, autoimmune diseases, etc. It could significantly help planning of tolerant treatment and increasing the quality of life of the patients.

In the present study, we try to clarify the relationship between the hydrophobicity, phase distribution and EPR signal reduction kinetic of several nitroxyl derivatives in biological systems in vitro and in vivo in the context of their application for tissue redox mapping using EPR. Some of these substances (spin-labeled nitrosoureas) combine both imaging characteristics and anticancer activity. The nitroxyl derivatives were characterized with different size and water-solubility. Our study was designed to investigate whether the level of hydrophobicity of the nitroxyl influences the mobility of free radical in the molecule, its reduction in the blood and its intracellular permeability - factors, determining the EPR signal reduction kinetic in living organism. The answer of this question allows a precise selection of most appropriate nitroxyls for evaluation of oxidation/reduction status of different biological specimens (blood, cells, or tissues) in vitro and in vivo. The EPR measurements were provided on isolated physiological fluids (e.g., saline solution, cell cultured medium, freshly isolated blood), as well as in the bloodstream of living anesthetized animals.

\section{Materials and Methods}

\section{Chemicals}

Carbamoyl-PROXYL (3-carbamoyl-2,2,5,5-tetramethylpyrrolidine-1-oxyl) and TEMPOL (4-hydroxy-2,2,6,6-tetramethyl-1-piperidin-1-oxyl) were purchased from Sigma-Aldrich. Nitroxyl-labeled nitrosoureas - SLENU (1-ethyl-3-[4-(2,2,6,6-tetramethylpiperidine-1-oxyl)]-1nitrosourea) and SLCNUgly (1-chloroethyl-3-[4-glycine(2,2,6,6-tetramethylpiperidine-1-oxyl)]-1-nitrosourea), were synthesized and purified according to Gadjeva et al. (1999, 2001), with slight modifications.

The structural formulas of nitroxyl derivatives are shown in Fig. 2. The octanol/phosphate buffered saline (PBS) partition coefficients $\left(\log \mathrm{P}_{\mathrm{oct}} / \mathrm{PBS}\right)$ were estimated at room temperature $\left(\sim 22-25^{\circ} \mathrm{C}\right)$.

Deionized water (deionization by the Milli-Q system) was used for all experiments. Other chemicals used were of analytical or HPLC grade.<smiles>CC1(C)CC(C(N)=O)C(C)(C)N1[O]</smiles>

Carbamoyl-PROXYL $\log P_{\text {octPBS }}:-0.158$<smiles>CCN(CC)C(=O)NC1CC(C)(C)N(C)C(C)(C)C1</smiles>

SLENU $\log P_{\text {octPBS: }}: 1.005$<smiles>CC1(C)CC(NC(=O)CCNC(=O)N(CCCl)C(=O)[O-])CC(C)(C)N1[O]</smiles>

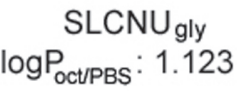

Figure 2. Structural formulas and octanol/PBS partition coefficients of nitroxyl derivatives. 


\section{Animals}

C57Bl/6 mice ( 6 to 8 weeks of age at the time of experiments; mean weight $\sim 25 \mathrm{~g})$ were used. All experiments were conducted in accordance with the guidelines of the Physiological Society of Japan and were approved by the Animal Care and Use Committee of the National Institute of Radiological Sciences (Chiba, Japan).

\section{In vitro EPR measurements}

The nitroxyl derivatives were first dissolved in DMSO to prepare $200 \mathrm{mmol} / \mathrm{l}$ stock solutions. These $200 \mathrm{mmol} / \mathrm{l}$ solutions were diluted with PBS, containing $1 \%$ bovine serum albumin (BSA) to prepare $2 \mathrm{mmol} / \mathrm{l}$ standard solutions. Each solution was put into a glass capillary and it's X-band EPR spectra was measured on X-band EPR instrument (JEOL, Akishima, Japan) with a TE-mode cavity. The capillary tube was positioned in the center of the TE-mode cavity using special sample holder. The measurements were made under the following conditions: microwave frequency $-9.4 \mathrm{GHz}$, magnetic field strength - $336 \mathrm{mT}$, microwave power - 2.0 $\mathrm{mW}$, field modulation frequency $-100 \mathrm{kHz}$, field modulation amplitude $-0.063 \mathrm{mT}$, time constant $-0.01 \mathrm{~s}$, sweep width - $10 \mathrm{mT}$, scan time (sweep time) $-1 \mathrm{~min}$.

In parallel, $2 \mu \mathrm{l}$ of $200 \mathrm{mmol} / \mathrm{l}$ stock solutions of nitroxyl derivative (in DMSO) were added to $198 \mu$ l freshly isolated blood (with heparin), bovine serum, or cell cultured medium (RPMI-1640), and EPR measurements were provided at the parameters mentioned above within 20-30 min scan-time.
The experimental error for repetitive measurements did not exceed $5 \%$.

\section{In vivo EPR measurements}

The experimental design is described in details by Zhelev et al. (2009). The mouse was anesthetized with $1.5 \%$ isoflurane using a face mask. The body temperature was kept within 36 $\pm 1^{\circ} \mathrm{C}$. The tail and jugular veins were cannulated by polyethylene tubes (PE10, Intramedic, Becton Dickinson Co.). The PE tube from the jugular vein was placed in the TE-mode cavity of EPR instrument (JEOL, Akishima, Japan). The end of the tube was connected to the syringe. All tubes and syringes were heparinized. The nitroxyls were injected intravenously through the tail vein $(0.4 \mu \mathrm{mol} / \mathrm{g}$ b.w. $)$. Immediately after the injection, the blood $(\sim 100 \mu \mathrm{l})$ was drained from the bloodstream through the jugular vein to reach the TE-mode cavity of EPR instrument. The EPR measurement was started. After finishing the measurement, the blood was infused back to the bloodstream of the mice. This cycle was repeated every 4-5 min within $30 \mathrm{~min}$, to register the EPR signal reduction kinetic of nitroxyl radical after its intravenous injection in the anesthetized animal. The EPR measurements were made under the conditions, mentioned in the previous paragraph.

\section{Results and Discussion}

Fig. 3A shows X-band EPR spectra of three piperidine derivatives (TEMPOL, SLENU and SLCNUgly) at differ-
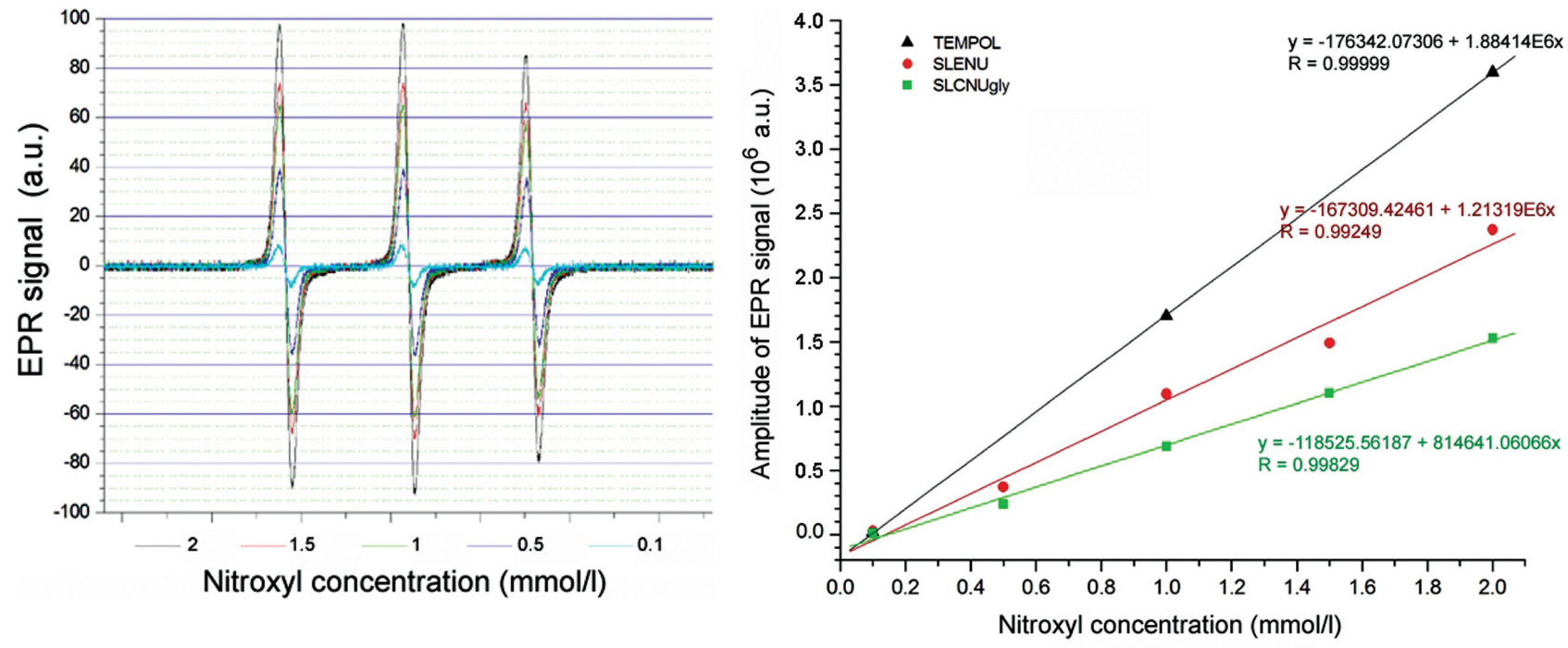

Figure 3. A. Typical X-band EPR spectra of SLENU at different concentrations in PBS (pH 7.4) containing 1\% BSA. The spectra are also representative for TEMPOL and SLCNUgly. B. Concentration-dependent curves of EPR signal enhancement of TEMPOL (green line), SLENU (red line), and SLCNUgly (black line) in PBS ( $\mathrm{pH}$ 7.4) containing 1\% BSA. Mean values from three independent experiments are given in the figure (SD values did not exceed 5\%). 
ent concentrations (from 0.1 to $2 \mathrm{mmol} / \mathrm{l}$ ). All nitroxyls showed sharp triplet lines without line-width broadening and marked anisotropy. They were dissolved in PBS, containing $1 \%$ BSA, and moved freely. The nitroxyls were distinguished in the EPR signal intensity, calculated as an integrated EPR spectrum. The EPR signal intensity increased linearly with increasing of nitroxyl concentration from 0.1 to $2 \mathrm{mmol} / \mathrm{l}$ (Fig. 3B). The EPR signal intensity increased also with increasing hydrophilicity of nitroxyls: $\mathrm{I}_{\text {SLCNUgly }}<\mathrm{I}_{\text {SLENU }}<$ ITEMPOL. Presumably, the strongly hydrophobic nitroxyls SLENU and SLCNUgly $\left(\log \mathrm{P}_{\text {oct/PBS }}\right.$ values were 1.005 and 1.123 , respectively) could form clusters, which will suppress (at least partially) the motion of nitroxyl radical due to steric restrictions and/or an enhanced viscosity of the sample, leading to a decrease in EPR signal intensity in PBS.

The different EPR signal enhancement of nitroxyls could be explained, at least partially, with different motion of nitroxyl radical depending on the substitutes at the $4^{\text {th }}$ position of the piperidine ring. Larger the substitute, slower the motion of nitroxyl radical, which results in lower EPR signal enhancement.

In blood samples in vitro, the EPR signal reduction kinetic was characterized with two phases: i) a significant decrease in the amplitude of EPR signal (in comparison with those in PBS) registered immediately (within 30-40 s) after addition of nitroxyl in freshly isolated blood; and ii) a subsequent slow decrease in the amplitude of EPR signal within 20-30 min incubation in blood (Fig. 4A). If the amplitude of EPR signal of nitroxyl in PBS was accepted as $100 \%$, it decreased immediately after its addition in blood ( 25, 55, or $65 \%$ for TEMPOL, SLENU, or SLCNUgly, respectively). This fast decay of EPR signal intensity in blood in comparison with PBS could be explained by two reasons: i) a fast reduction of nitroxyl radical to hydroxylamine from the blood reducing equivalents; and ii) an interaction of nitroxyl derivatives with blood cells and/or lipoproteins and restriction of the motion of nitroxyl radical. Both events are accompanied with reduction of EPR signal intensity. The hydrophobic nitroxyls SLENU and SLCNUgly will characterize by fast and tight incorporation in cell membranes, while the hydrophilic TEMPOL will exist predominantly in the aqueous phase of the blood, moving freely and easily.

Figure 4. A. Time-decay of EPR signal amplitude after addition of nitroxyl derivative $(2 \mathrm{mmol} / \mathrm{l})$ in freshly isolated blood. Mean values from three independent experiments are given in the figure (SD values did not exceed 10\%). B. Dynamics of EPR signal intensity in the blood stream of live mice under anesthesia after intravenous (i.v.) injection of nitroxyl derivative $(0.4 \mu \mathrm{mol} / \mathrm{g}$ b.w.). Mean values from three independent experiments are given in the figure (SD values did not exceed 30\%). C. Hypothetical mechanism for decreasing of EPR signal intensity of nitroxyl derivatives in blood. $t$, incubation time; $\mathrm{t}_{\mathrm{i}}$, time after i.v. injection.
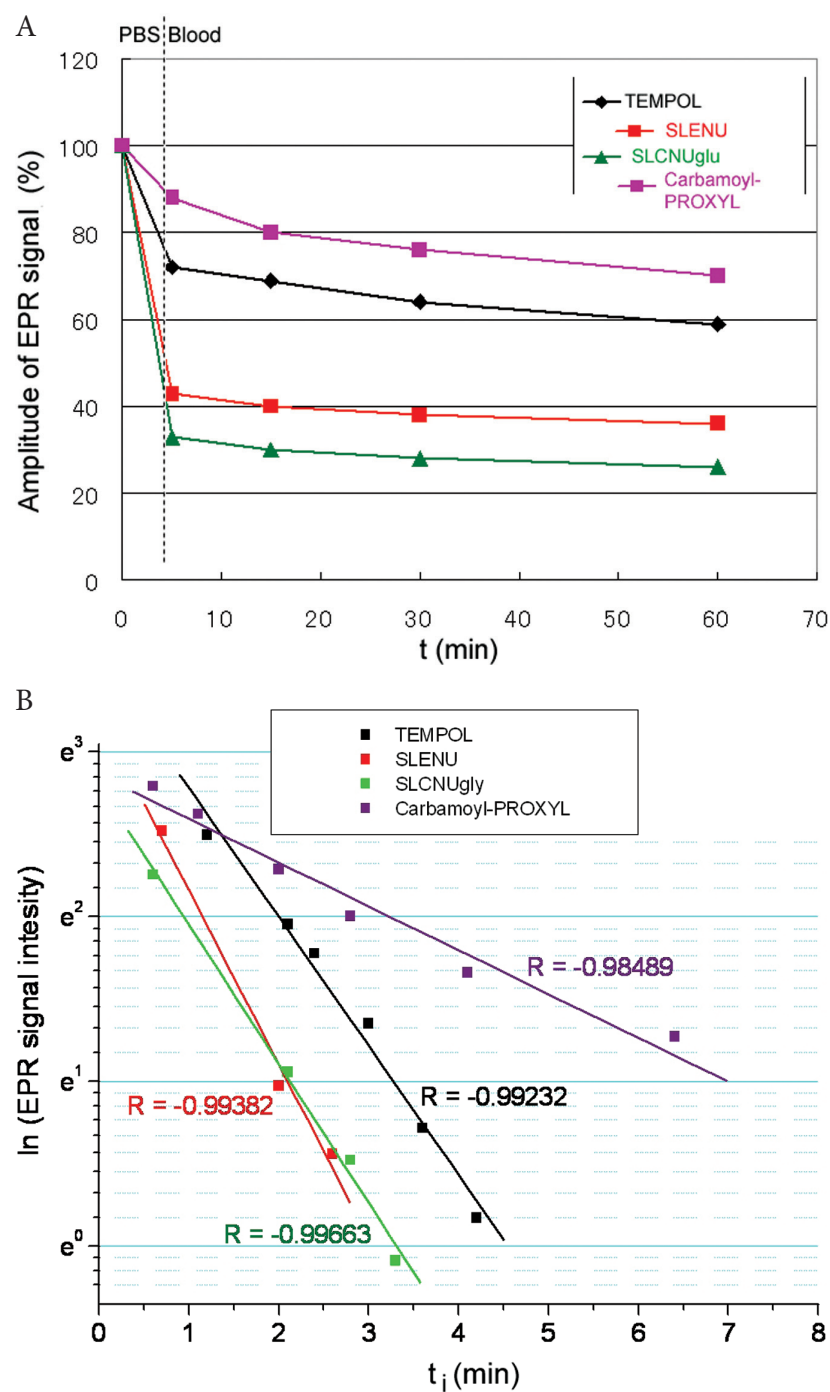

C

BLOOD PLASMA

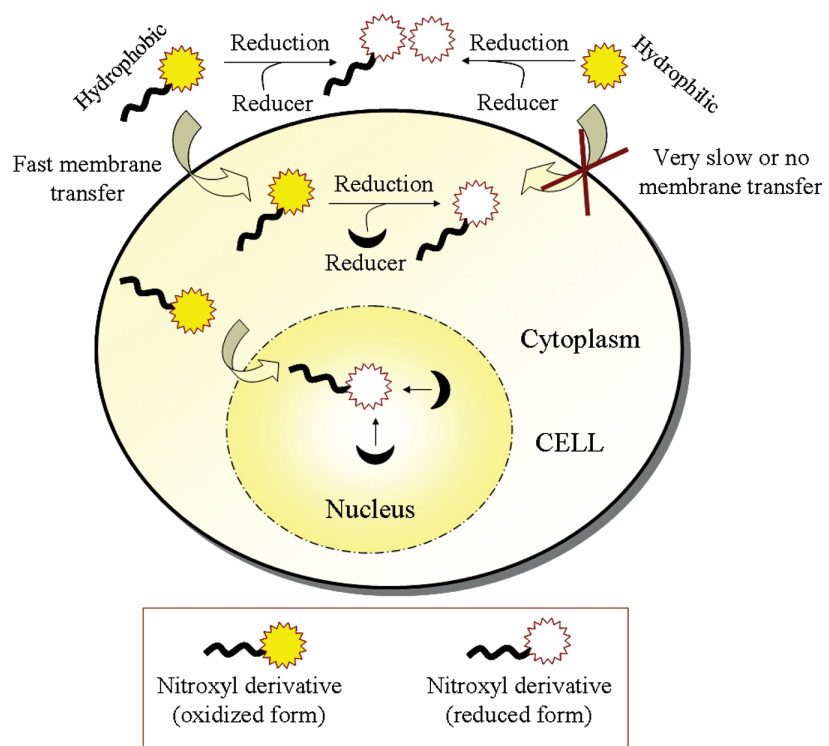


In EPR data analysis of nitroxyl radicals, the variations in Line 3 in the triplet of EPR spectrum (and especially Line 2/Line 3 amplitude ratio) is considered as an indicator for the motion of nitroxyl radical (Matsumoto et al. 2007). If the ratio increases, the motion decreases. Table 1 shows the analysis of rotational correlation times $\left(\tau_{\mathrm{c}}\right)$ of nitroxyl derivatives in PBS and blood. This parameter was calculated by the method, described in Hayashi et al. (1992) and Zhelev et al. (2009).

In PBS and blood, the rotational correlation time increased with increasing hydrophobicity of nitroxyl derivatives, increasing of molecular volume and reducing of segmental motion of piperidine ring inside of the molecule: $\tau_{\mathrm{c}}^{\text {TEMPOL }}<\tau_{\mathrm{c}}^{\text {SLENU }}<\tau_{\mathrm{c}}^{\text {SLCNUgly }}$. $\tau_{\mathrm{c}}$ values are higher in blood than in PBS. The data suggest that the motion of nitroxyl radical in blood decreased for each nitroxyl, regardless of its hydrophobicity. Presumably, the interaction of nitroxyls with blood cells and/or lipoproteins is the most likely mechanism for decreasing of their EPR signal intensity in blood. In serum and cell cultured medium, in the absence of cells and in negligible amount of reducing equivalents, we did not observe significant changes in EPR spectrum profile and EPR signal reduction kinetic in comparison with PBS (Table 1), which is an indirect proof for the hypothesis, described above.

The slow decrease of EPR signal intensity in Fig. 4A could be explained by the trans-membrane transfer (flip-flop) of nitroxyl derivative through cell membranes (from the bloodstream into the cells) and its subsequent reduction to hydroxylamine from the cellular reducing equivalents (Fig. 4C). Since the spontaneous flip-flop is usually a slow process, we detected a slow decrease of EPR signal intensity - about $10 \%$ within $20-30 \mathrm{~min}$ incubation in blood.

In vivo, a similar tendency was observed. The kinetic curves of EPR signal reduction of nitroxyl derivatives in the bloodstream of living mice are shown in Fig. 4B. They were characterized with two phases: i) an increase

Table 1. Variations in the rotational correlation time $\left(\tau_{c}\right)$ of several nitroxyls in PBS and blood

\begin{tabular}{|l|c|c|c|c|}
\hline \multirow{2}{*}{ Nitroxyl } & \multicolumn{4}{|c|}{$\tau_{\mathrm{c}}\left(\times 10^{-12} \mathrm{~s}\right)$} \\
\cline { 2 - 5 } & PBS & medium & serum & blood \\
\hline TEMPOL & 10.56 & 10.51 & 10.48 & 20.33 \\
\hline SLENU & 29.45 & 29.38 & 29.31 & 32.87 \\
\hline SLCNUgly & 31.38 & 31.40 & 31.25 & 46.20 \\
\hline
\end{tabular}

All calculations are made from the EPR spectra of nitroxyls $(2 \mathrm{mmol} / \mathrm{l})$ in PBS or freshly isolated blood from mouse (after 5 min incubation). The data are mean from three independent experiments (SD did not exceed 5\%). All values are approximated, calculated on the basis of A-value for TEMPO. of EPR signal intensity within $30 \mathrm{~s}$ after nitroxyl injection - mainly as a result of increasing of nitroxyl concentration in the bloodstream (not shown); and ii) a decrease of EPR signal intensity within 1-5 min after the injection of nitroxyl, presumably as a result of its reduction in the blood plasma, transportation from the bloodstream to the cells and tissues, and further reduction from the cell/tissue reducing equivalents (Fig. 4B,C). The time-decay of EPR signal intensity in vivo correlated with the hydrophobicity of nitroxyl derivative and its cell permeability. The rate of time-decay was slower for the hydrophilic TEMPOL and faster for the hydrophobic SLENU and SLCNUgly. Since all investigated nitroxyls were cell permeable, the EPR signal disappeared completely within 4-5 min after their injection. To approve this assumption, we used carbamoylPROXYL. Carbamoyl-PROXYL is a pyrrolidine derivative, which is characterized with high water-solubility and low permeability for cell membranes (Matsumoto et al. 2006, 2007). We observed that the time-decay of EPR signal of carbamoyl-PROXYL in the bloodstream was much slower than for other nitroxyls (TEMPOL, SLENU, SLCNUgly), presumably, as a result of slower transportation of this compound to the cells and tissues.

We established that the spin-labeling of nitrosourea did not affect negatively its anticancer activity. The optimal anticancer activity of non-labeled $\mathrm{N}$-(2-chloroethyl)$\mathrm{N}$-cycloxehyl-N-nitrosourea (CCNU) and spin-labeled nitrosoureas (SLENU and SLCNUgly) on mice with experimental lymphoma was observed at 20, 45 and $45 \mathrm{mg} / \mathrm{kg}$ b.w., respectively. The spin-labeling decreased the median letal dose (LD50) of nitrosoureas. In mice, the LD50 for CCNU, SLENU, and SLCNUgly was 56, 100, and $125 \mathrm{mg} / \mathrm{kg}$ b.w., respectively (Zhelev et al. 2009). For comparison, LD50 of TEMPOL was $210 \mathrm{mg} / \mathrm{kg}$ b.w.

The nitroxyl radicals could also serve as regulators of reduction/oxidation balance in cancer and normal tissues. It is well-known that the cancer cells and tissues are characterized with a high level of reducing equivalents (necessary for cell proliferation and immortalization) and a very high resistance to oxidation (Kagan et al. 1990; Trachootham et al. 2008). In contrast, the normal tissues of cancer-bearing organism are characterized with a decreased level of reducing equivalents, an enhanced level of reactive oxygen species (ROS) and a high susceptibility to oxidative stress (Kagan et al. 1990; Trachootham et al. 2008). Our study suggests that the nitroxyl-labeling of anticancer drug could increase its specific anticancer effect, presumably by decreasing the level of reducing equivalents and/or free radicals in the tissues of cancer-bearing animals (Fig. 5). In the normal tissues of cancer-bearing mammalians, the nitroxyl radical will serve predominantly as a free radical scavenger and the balance will be extended to the following direction: nitroxyl radical $->$ oxoammonium 


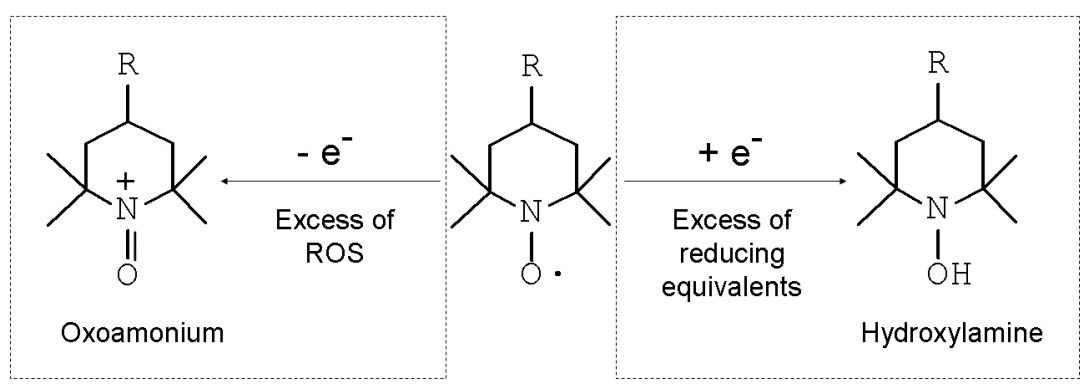

Figure 5. Nitroxyl radical as a regulator of tissue reduction/oxidation balance - dual activity.

(Fig. 5). A recent study of Gadjeva et al. (2007) supports this assumption.

\section{Conclusion}

The data demonstrate that the hydrophobicity of nitroxyl derivatives, which determines their phase partition and distribution in cells, can play a crucial role for their consideration as potential candidates for tissue redox mapping using EPR. Our study suggests that the hydrophobic cellpermeable nitroxyl derivatives seem most appropriate for evaluation of cell and tissue oxidation/reduction status, while the hydrophilic nitroxyls (impermeable for cell membranes or with very slow cell permeability) are most appropriate for evaluation of oxidation/reduction status of blood using EPR imaging. Since the nitroxyl radicals possess a comparatively high longitudinal relaxation time $\mathrm{T}_{1}$ (Hyodo et al. 2006, 2008), the described data are also useful for evaluation of blood and tissue oxidation/reduction status, using magnetic resonance imaging (Zhelev et al. 2009).

It is widely accepted that pyrrolidine derivatives are most appropriate for tissue redox mapping than piperidine derivatives because of long-lived EPR signal intensity in biological samples (Kocherginsky et al. 1995). However, this could provoke misinterpretations if cell permeability and distribution of nitroxyl derivatives is neglected. To express their redox sensing effect in the tissues, the nitroxyls have to be delivered into the cells and to access the intracellular reducing equivalents. If pyrrolidine derivatives do not enter the cells, they have to be considered as inappropriate candidates for tissue redox mapping using EPR.

The described data demonstrate also the potential advantage of nitroxyl labeling of conventional anticancer drugs for image-guided drug delivery. Since the nitroxyl radicals are comparatively low toxic and harmless for normal tissues of healthy mammalians (Shacter et al. 2000; Metz et al. 2004; Ravizza et al. 2004; Gariboldi et al. 2006), the nitroxyl-label- ling could be considered as environmentally friendly and with minimal risk of side-effects.

Acknowledgments. This study was supported in part from bilateral contract between NIRS (Chiba, Japan) and Trakia University (Stara Zagora, Bulgaria).

\section{References}

Gadjeva V., Raikov Z. (1999): Synthesis and antitumor activity of 4-[N'-[N-(2-chloroethyl)-N-nitrosocarbamoyl] hydrazono]-2,2,6,6-tetramethylpiperidine-1-oxyl. Pharmazie 54, 231-232

Gadjeva V., Koldamova R. (2001): Spin-labeled 1-alkyl-1-nitrosourea synergists of antitumor antibiotics. Anticancer Drug Des. 16, 247-253

Gadjeva V., Tolekova A., Vasileva M. (2007): Effect of the spin-labelled 1-ethyl-1-nitrosourea on CCNU-induced oxidative liver injury. Pharmazie 62, 608-613

Gariboldi M. B., Terni F., Ravizza R., Meschini S., Marra M., Condello M., Arancia G., Monti E. (2006): The nitroxide Tempol modulates anthracycline resistance in breast cancer cells. Free Radic. Biol. Med. 40, 1409-1418; doi:10.1016/j.freeradbiomed.2005.12.011

Hayashi H., Iwasaki T., Onodera Y., Nagase T., Itabashi O. (1992): Proceedings of Tohoku Kogyo Gijitsu Shikenjo Houkoku. pp. 29-33 (in Japanese)

Hirayama A., Yoh K., Nagase S., Ueda A., Itoh K., Morito N., Hirayama K., Takahashi S., Yamamoto M., Koyama A. (2003): EPR imaging of reducing activity in Nrf2 transcriptional factor-deficient mice. Free Radic. Biol. Med. 34, 1236-1242; doi:10.1016/S0891-5849(03)00073-X

Hyodo F., Matsumoto K., Matsumoto A., Mitchell J. B., Krishna M. C. (2006): Probing the intracellular redox status of tumors with magnetic resonance imaging and redoxsensitive contrast agents. Cancer Res. 66, 9921-9928; doi:10.1158/0008-5472.CAN-06-0879

Hyodo F., Chuang K. H., Goloshevsky A. G., Sulima A., Griffiths G. L., Mitchell J. B., Koretsky A. P., Krishna M. G. (2008): Brain redox imaging using blood-brain barrier-permeable nitroxide MRI contrast agent. J. 
Cereb. Blood Flow Metab. 28, 1165-1174; doi:10.1038/ jcbfm.2008.5

Kagan V. E., Bakalova R. A., Karakashev P. E. (1991): Lipid peroxidation in the tumor cells and tissues of tumor-bearing animals. In: Membrane Lipid Oxidation (Ed. C. VigoPelfrey), pp. 191-208, CRC Press, Boca-Raton, Florida

Kocherginsky N., Swartz H. M. (1995): Chemical reactivity of nitrooxides. In: Nitroxide Spin Labels, Reactions in Biology and Chemistry, CRC Press, Boca-Raton, Florida

Kuppusamy P., Li H., Ilangovan G., Cardounel A., Zweier J., Yamada K., Krishna M., Mitchel J. (2002): Noninvasive imaging of tumor redox status and its modification by tissue glutathione levels. Cancer Res. 62, 307-312

Matsumoto K., Hyodo F., Matsumoto A., Koretsky A. P., Sowers A. L., Mitchell J. B., Krishna M. C. (2006): High-resolution mapping of tumor redox status by magnetic resonance imaging using nitroxides as redox-sensitive contrast agents. Clin. Cancer Res. 12, 2455-2462; doi:10.1158/1078-0432. CCR-05-2747

Matsumoto K., Narazaki M., Ikehira H., Anzai K., Ikota N. (2007): Comparisons of EPR imaging and T1-weighted MRI for efficient imaging of nitroxyl contrast agents. J. Magn. Res. 187, 155-162; doi:10.1016/j.jmr.2007.03.013

Metz J. M., Smith D., Mick R., Lustig R., Mitchell J., Cherakuri M., Glatstein E., Hahn S. M. (2004): A phase I study of topical Tempol for the prevention of alopecia induced by whole brain radiotherapy. Clin. Cancer Res. 10, 6411-6417; doi:10.1158/1078-0432.CCR-04-0658

Ravizza R., Cereba E., Monti E., Gariboldi M. B. (2004): The piperidine nitroxide Tempol potentiates the cytotoxic effects of temozolomide in human glioblastoma cells. Int. J. Oncol. $25,1817-1822$
Shacter E., Williams J. A., Hinson R. M., Sentürker S., Lee Y. J. (2000): Oxidative stress interferes with cancer chemotherapy: inhibition of lymphoma cell apoptosis and phagocytosis. Blood 96, 307-313

Trachootham D., Lu W., Ogasawara M. A., Nilsa R. D., Huang P. (2008): Redox regulation of cell survival. Antioxid. Redox Signal. 10, 1343-1374; doi:10.1089/ars.2007.1957

Utsumi H., Yamada K., Ichikawa K., Sakai K., Kinoshita Y., Matsumoto S., Nagai M. (2006): Simultaneous molecular imaging of redox reactions monitored by Overhauserenhanced MRI with $14 \mathrm{~N}$ - and $15 \mathrm{~N}$-labeled nitroxyl radicals. Proc. Natl. Acad. Sci. U.S.A 103, 1463-1468; doi:10.1073/pnas.0510670103

Valgimigli L., Pedulli G. P., Paolini M. (2001): Measurement of oxidative stress by EPR radical-probe technique. Free Radic. Biol. Med. 31, 708-716; doi:10.1016/S08915849(01)00490-7

Yokoyama H., Sato T., Fukui K., Itoh O., Ohya H., Akatsuka T. (2004): Noninvasive in vivo determination of intracerebral oxygen concentration in rats by a longitudinally detected ESR (LODESR) technique. Magn. Res. Med. 51, 595-601; doi:10.1002/mrm.10725

Zhelev Z., Bakalova R., Matsumoto K., Aoki I., Gadjeva V., Anzai K., Kanno I. (2009): Nitroxyl radicals for labeling of conventional therapeutics and noninvasive magnetic resonance imaging of their permeability for blood-brain barrier: relationship between structure, blood clearance, and MRI signal dynamic in the brain. Mol. Pharm. 6, 504-512; doi:10.1021/mp800175k

Received: January 10, 2009

Final version accepted: July 1, 2009 